\title{
Aberration-free imaging by coherent diffractive imaging
}

\author{
J.S.Wu*, J.C.H. Spence and U. Weierstall \\ Department of Physics, Arizona State University, Tempe, AZ 85287. USA. (jinsong.wu@asu.edu)
}

Experimental demonstrations of diffractive imaging using electrons [1,2] and X-rays [3] have appeared over the last decade. Here images are reconstructed directly from diffraction patterns by numerical processing, using a computer to replace the image-forming lens. The amplitudes in reciprocal space are measured directly from the diffraction patterns. The iterative algorithms used to solve this non-crystallographic phase problem for nonperiodic objects are based mainly on work by Gerchberg, Saxton, Fienup and others $[4,5]$. In principle the resulting images are diffraction-limited and aberration free. The first atomic-resolution image reconstructed from a diffraction pattern showed a single double-walled nanotube [2]. In the work, the nanotube spanned a small hole in a carbon film, in order to limit the background contribution from any supporting substrate film. Recently we developed a general method for applying diffractive imaging to small objects supported by a thin film in the transmission geometry. In this work, we have recorded the pattern from the carbon film separately nearby, and modified both the Fienup Hybrid input-output algorithm, and the remarkable flipping algorithm of Oszlányi and Süto [6-7] to take this into account [1]. The average of the weak carbon film background has been subtracted, and difficulties in sample preparation for diffractive imaging are avoided.

We use a JEOL 2010F electron microscope with a field-emission gun to collect the nano-diffraction patterns with spatial coherence spanning the particle. To obtain diffraction data of sufficient quality and dynamic range to use quantitatively, it was neccessary to use image plates for recording, to avoid CCD blooming. Illuminated by a coherent nano-probe with diameter around 40-50 nm, nanodiffraction patterns were taken from the nano-gold particle as well as the carbon film nearby. Figure 1 shows the nano-gold particle illuminated by the nano-probe. Figure 3 shows the amplitudes of the reconstructed exit-wave of the nano-gold particle reconstructed by this method at sub-angstrom resolution of $0.9 \AA$ from the electron diffraction pattern shown in figure $2 a$. Note the inset elastic diffuse "shape transform" scattering from the icosohedral particle (fig.2b) laid down around the Bragg beam at $b$. In figure 4, which a portion of fig. 3 at higher magnification, we show the image of planar defects in the gold nanoparticle. The coherent diffractive imaging technique provides an aberration-free environment for structural determination. The resolution of the images in the electron microscopy case is limited by temperature factors and noise. Use of the TEM also has the great advantage that a medium-resolution image may be used for the support estimate.

\section{References}

[1] J.S. Wu, U. Weierstall and J.C.H. Spence, Nature Materials 4 (2005) 912.

[2] J.M. Zuo, I.A. Vartanyants, M. Gao, M. Zhang, L.A. Nagahara, Science, 300 (2003) 1419.

[3] H. Chapman, A. Barty, T. Beetz, C. Cui, H. He, M. Howells, S. Marchesini, A. Noy, R. Rosen, J.C.H. Spence, U. Weierstall, J. Opt Soc. Am. 23 (2006) in press.

[4] J. C.H.. Spence, in "Science of Microscopy". Eds. P. Hawkes and J. Spence. Springer, (2006). in press.

[5] Stark, H. Ed. Image Recovery. Theory and applications. Academic Press. (1987). New York.

6] G. Oszlányi and A. Süto, Acta Cryst. A60 (2004) 134-141.

7] J.S. Wu, J.C.H. Spence, M. O’Keeffe, and T. Groy, Acta Cryst. A60 (2004) 326-330. 
[8] The work is supported by ARO award W911NF-05-1-0152.

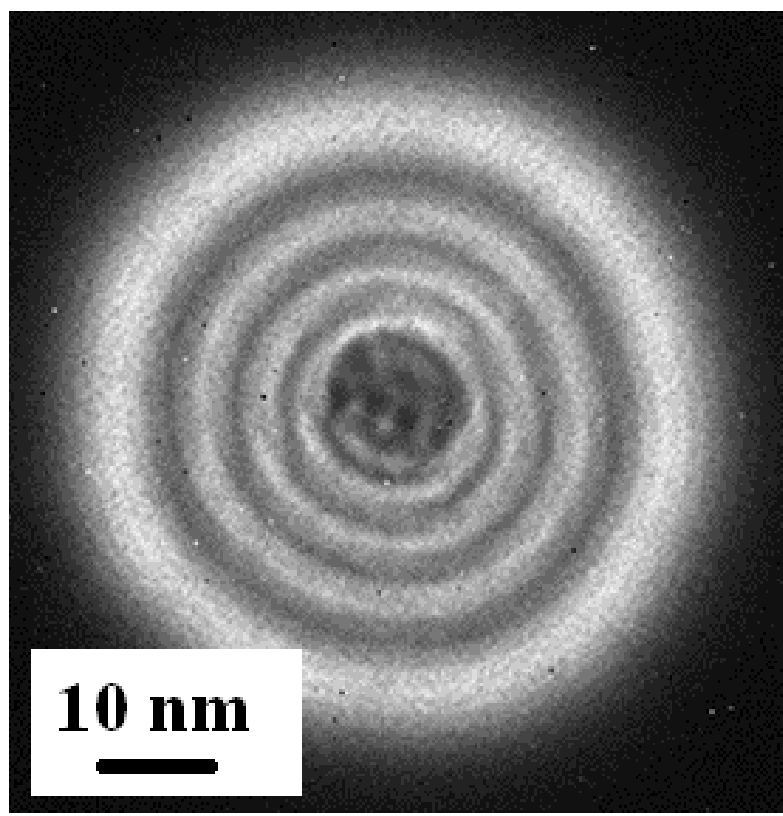

Figure. 1. TEM image of the nano-gold particle illuminated with a parallel electron nano-probe.

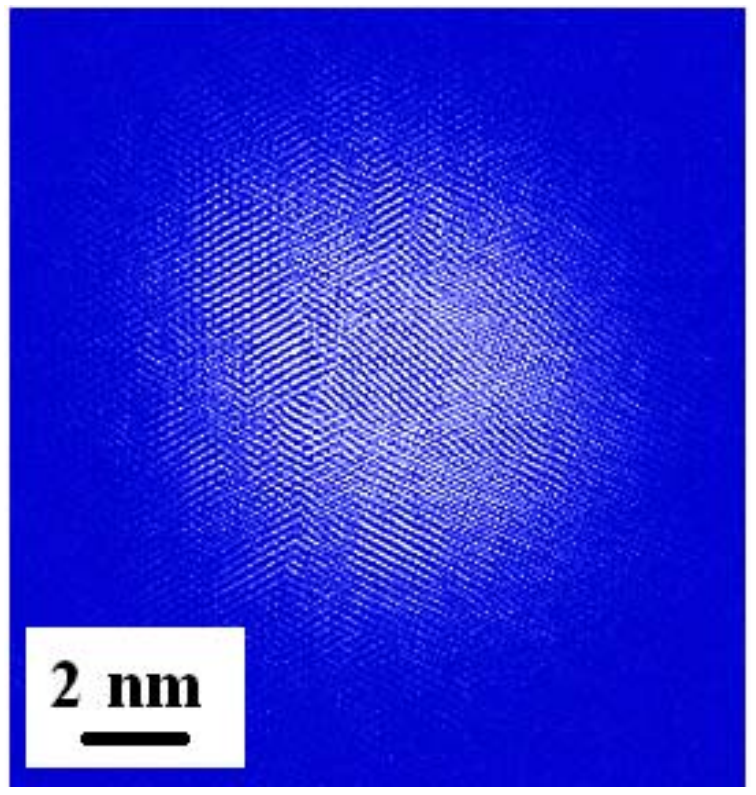

Figure. 3. Amplitudes of the reconstructed exit-wave of the nano-gold particle.

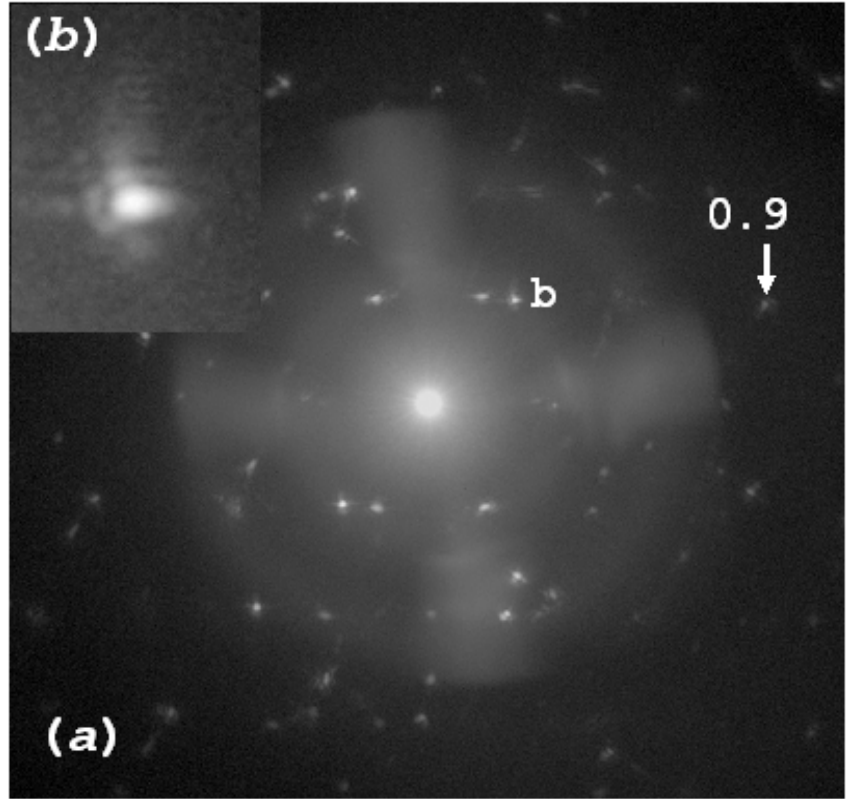

Figure 2. (a) Diffraction pattern from gold ball recorded on image plate. (b) "shape transform" directly from the diffraction pattern (a) by around Bragg spot b. JEOL 2010 coherent SAD mode.

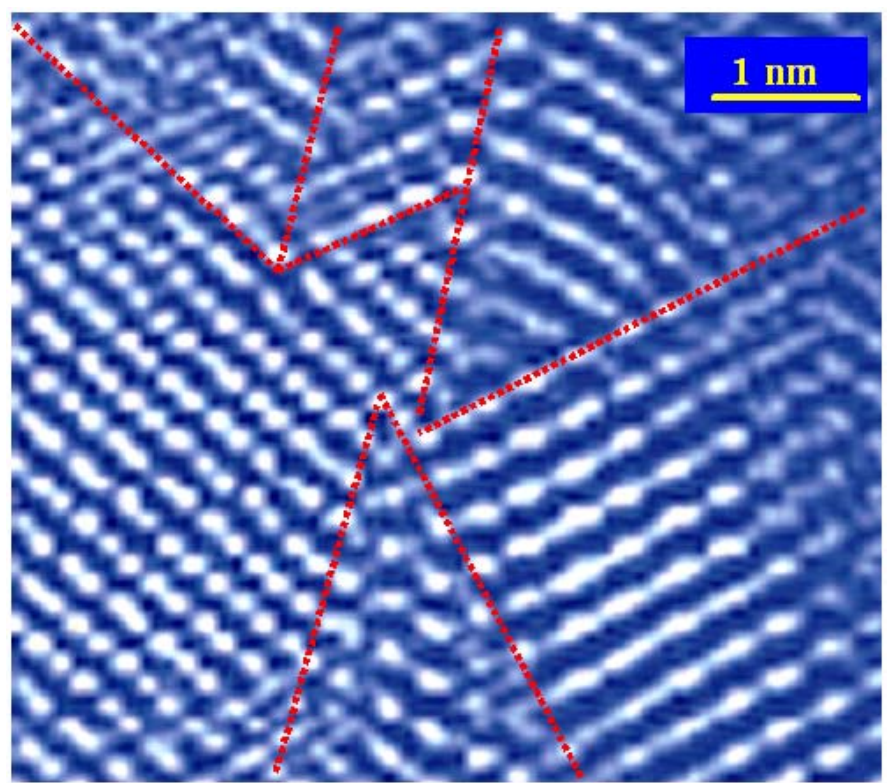

Figure 4. This is not an HREM image ! It was obtained directly from the diffraction pattern in figure 2 by computational solution of the phase problem for every pixel in the diffraction pattern. Note planar defects. 